\title{
Lectures Critiques
}

Stéphane Baele

The Deleuge Dictionary

Parr A., Edinburgh, Edinburgh University Press, $2005^{1}$

"Why a Deleuze dictionary? it might seem a particularly craven, disrespectful, literal-minded and reactive project to form a Deleuze dictionary. " C'est par cette question et ce constat de paradoxe apparent que Claire Colebrook ouvre le premier dictionnaire consacré à la conceptuelle du philosophe français Gilles Deleuze. C’est qu'effectivement, tant la pensée de l'auteur de Nietæssche et la philosophie ou du diptyque hydresque Capitalisme et Schizophrénie se fonde sur le principe d'une ontologie de la différence et de la mobilité, tant sa forme a emprunté les détours parfois abscons imposés par le choix affirmé d'une adéquation entre épistémologie et ontologie, qu’il semblait impossible et surtout traitre de fixer et enfermer le mouvement deleuzien dans un dictionnaire, qui, après tout, est censé édicter l'immuable.

Editeur du Delenze Dictionary, Adrian Parr a relevé le défi imposé par cette apparente contradiction. En s'entourant d'une vingtaine d'auteurs, dont de nombreux Australiens mais surtout sans aucun francophone, il a su créer les conditions de réussite de son projet. Non seulement le format des définitions (de courts articles d'une ou deux pages, avec indication des liens vers d'autres sujets proches, se présentent parfois sous la forme d'additions - comme par exemple l'entrée «control society + state theory»), mais aussi l'impressionnante conscience de l'esprit deleuzien - plus impérative encore que la connaissance analytique des textes - démontrée par les auteurs, déjouent le piège du statisme. Avec une introduction de six pages en véritable pamphlet indirect contre les adeptes de l'épistémologie philosophique unique, avec un ensemble d'articles hétéroclites de grande qualité unis par une conscience commune forte, et dans un style accessible, force est d'avouer que cet ouvrage confirme la vitalité de la particulière et parfois ostracisée école «postmoderne » anglo-saxonne, et plus précisément australienne. D’une seule voix, le collectif publié ici par les Presses Universitaires d'Edinburgh met à la disposition des lecteurs de Deleuze - débutants comme expérimentés - un ouvrage de référence, dont le dernier des mérites n'est sans doute pas d'avoir du même coup proposé un système enfin cohérent de traduction du français vers l'anglais. On remarquera aussi, dans la foulée, les ouvrages corollaires de ce

\footnotetext{
${ }^{1}$ Et : Bonta M., Protolevi J., Deleuze and Geophilosophy. Edinburgh: Edinburgh University Press, Deleuze Connections, 2006.

Et : Fuglsang M., Sorensen B.M., ed., Deleuze and the Social, Edinburgh: Edinburgh University Press, Deleuze Connections, 2006.
} 
dictionnaire, tous parus aux Edinburgh University Press sous la collection unique «Deleuze Connections", comme par exemple Deleuze and the Social, ou Deleure and Geophilosophy, et qui font montre des mêmes qualités que l'ouvrage édité par Adrian Parr.

\section{Stéphane Baele}

Searl and Foucault on Truth

Prado C.G., New-York, Cambridge University Press, 2006

Pour une majorité des auteurs académiques, explorer les présupposés mêmes de sa tradition de pensée, pour les remettre en question, est une démarche qui n'est pas à l'ordre du jour. Et a fortiori, lorsqu'il s'agit de s'interroger sur les dynamiques de ce qui peut être devenu un dogme, en l'occurrence son appartenance tranchée à l'une des deux traditions qui divisent le monde philosophique d'aujourd'hui - à savoir la pensée « continentale » et l'école « analytique » le plus souvent anglo-saxonne -, on pourrait prétendre que la mise en route elle-même est devenue structurellement impossible. Puisque les présupposés fondateurs de ces deux approches s'opposent diamétralement, comment - et surtout finalement pourquoi - encore y chercher une réconciliation?

C’est précisément parce que ce thème du rapprochement est trop rare, qu'il faut éviter de laisser passer inaperçu un ouvrage qui s'y attelle sérieusement. Face à ce qui est devenu un problème d'« intolérance » et de "stéréotypes », C.G. Prado se met en quête de montrer que l'opposition entre la philosophie analytique et continentale n'est que le résultat d'une histoire d'incompréhension mutuelle. Car sans doute diabolise-t-on l'adversaire, qui finalement se pose les mêmes questions et parfois même propose des réponses compatibles, sinon semblables. Déjà auteur d'un intéressant livre sur la question ( $A$ House Divided), Prado propose ici d'opposer puis de rassembler deux icônes de leurs traditions respectives sur une question commune, pour montrer que l'investigation philosophique ne gagne rien à rester dans ses « tranchées ».

Ce seront Searle et Foucault, sur le problème de la vérité («truth»). Par une analyse parallèle puis croisée, l'auteur examine les deux interprétations apparemment opposées du jugement vrai. Son analyse rigoureuse et claire, ses rappels aux idées fondamentales des deux auteurs, donnent un essai éthiquement nécessaire. Néanmoins, il convient de s'interroger sur la pertinence du choix du thème de la vérité comme terrain de confrontation. Bien sur, il s'agit d'un thème ouvertement abordé par les deux auteurs, et traité effectivement de manière opposée ; mais Foucault n'a jamais sombré dans les travers du relativisme béat, et comme Han l'avait déjà 
interprété, son acceptation de la notion de vérité pouvait presque être vue comme indépendante de la réalité construite. Dès lors, le thème de la vérité comme rapport de correspondance au monde est chez lui écarté au profit de celui de la nécessaire construction de ce rapport, et le débat avec Searle devrait devenir celui de l'historicité ou non des processus constituant notre rapport au monde. C'est à ce débat-là qu'il aurait été intéressant de consacrer un chapitre de plus en fin de livre, après avoir effectivement compris que le les deux auteurs auraient pu se comprendre sur l'issue de l'existence de la réalité - qui n'est pas la même que celle de la vérité.

Finalement, Prado aura mis face-à-face Foucault et Searle sur la question de la réalité, et aura peut-être de ce fait évité le principal objet de dissension entre continentaux et analytiques : celui de la structure, de la texture, de l'aspect même de cette réalité, et subséquemment celui de la véritable question de l'établissement de la vérité - historique ou universelle. Bien sur, choisir Derrida ou Baudrillard n'aurait pas fait grand sens, mais on attends toujours une confrontation ontologique entre les deux écoles, puisqu'il semble de fait évident que Searle et Foucault ne sont pas les plus durs représentants des deux écoles en question. En somme, si l'attrait du livre de Prado est avant tout éthique, son contenu n'en est pas moins solide mais n'apporte pas encore la clef ouvrant ou fermant définitivement la porte entre les deux écoles. Y en a-t-il seulement une ? Rien n'est moins sur, mais il devient nécessaire de se comporter comme si, au lieu de céder à la prétention de la voie royale puisque celle-ci est impossible. A moins que ceci ne soit une assertion typiquement « continentale ». 